\title{
Jean-Louis Rastoin et Gérard Ghersi - Le système alimentaire mondial : concepts et méthodes, analyses et dynamiques - Éditions Quæ, 565 p., 45 euros
}

\section{Gérard Azoulay}

\section{(2) OpenEdition}

\section{Journals}

Édition électronique

URL : http://journals.openedition.org/economierurale/3444

DOI : 10.4000/economierurale.3444

ISSN : 2105-2581

Éditeur

Société Française d'Économie Rurale (SFER)

Édition imprimée

Date de publication : 30 mai 2012

Pagination : 98-99

ISSN : 0013-0559

Référence électronique

Gérard Azoulay, « Jean-Louis Rastoin et Gérard Ghersi - Le système alimentaire mondial : concepts et méthodes, analyses et dynamiques - Éditions Quæ, 565 p., 45 euros », Économie rurale [En ligne], 329 | mai-juin 2012, mis en ligne le 30 mai 2012, consulté le 21 septembre 2020. URL : http://

journals.openedition.org/economierurale/3444 ; DOI : https://doi.org/10.4000/economierurale.3444 
Jean-Louis Rastoin et Gérard Ghersi

\section{Le système alimentaire mondial Concepts et méthodes, analyses et dynamiques}

Éditions Quæ, 565 p., $45 €$

a situation alimentaire mondiale s'est -aggravée depuis la dernière crise des prix alimentaires des années 2007-2008 qui a sensiblement accru le nombre de personnes souffrant des diverses manifestations de la faim. L'analyse des facteurs tant conjoncturels que structurels de cette crise renvoie fondamentalement au mode de fonctionnement actuel de l'économie agricole mondiale. La forte croissance de la production alimentaire des cinquante dernières années n'a pas mis fin au problème de la faim. Les disponibilités alimentaires sont suffisantes pour nourrir la population mondiale. Depuis 1961, la production céréalière mondiale a triplé alors que la population a doublé. Mais il est impossible pour environ $15 \%$ des habitants de la planète de produire de la nourriture ou d'y avoir accès. Ce paradoxe résulte d'une forme spécifique d'organisation de l'économie agricole mondiale qui tolère que plus de 960 millions de personnes des pays du Sud, mais aussi des sociétés d'abondance, ne satisfassent pas de manière adéquate leurs besoins nutritionnels.

L'ouvrage de Jean-Louis Rastoin et de Gérard Ghersi décrit et analyse les multiples dimensions du système alimentaire mondial qui produit cette situation paradoxale. L'ouvrage constitue à ce titre une somme de plus de 550 pages qui se présente sous la forme d'un traité académique dont l'ambition est d'exposer les concepts, les théories et les méthodes de l'analyse du système alimentaire, système que Louis Malassis` définissait comme « la

1. Louis Malassis, Nourrir les hommes, Paris, Flammarion, 1993, $126 \mathrm{p}$. manière dont les hommes s'organisent, dans l'espace et dans le temps, pour consommer et obtenir leur nourriture ».

L'ensemble des outils d'analyse du système est présenté depuis les bases de l'analyse systémique, les instruments de la comptabilité nationale et de l'analyse des filières permettant d'analyser les relations économiques qui contribuent à la production alimentaire, jusqu'aux déterminants de la consommation alimentaire. L'analyse du système alimentaire, système complexe, est menée en y introduisant les acteurs et leurs interactions, la concentration des industries et le pouvoir de négociation des acteurs, les processus de régulation et les systèmes d'information.

Les mécanismes d'internationalisation, de globalisation font l'objet d'une analyse tant théorique qu'empirique, alliant les mouvements de produits agricoles, les marchés, leur instabilité et les discussions menée à l'Organisation mondiale du commerce (OMC). L'impact de ces instabilités des prix des produits agricoles constitue à l'évidence une préoccupation majeure des Pays à faible revenu et à déficit vivrier (PFRDV). La conséquence au niveau microéconomique de la récente crise des prix est une modification de la consommation des plus pauvres, dont les régimes alimentaires se trouvent modifiés dans leur composition quand la substitution entre biens similaires mais de prix différents reste possible. Lorsque ce n'est pas le cas, la baisse de la consommation des produits non alimentaires ne suffit souvent pas, lorsque le coefficient budgétaire alimentaire est élevé, à éviter la sousalimentation (alors souvent accompagnée d'émeutes de la faim). La dépendance extérieure est telle, dans ces pays, que se pose nécessairement la question des politiques commerciales alternatives à une ouverture généralisée imposée plus par les accords avec la Banque mondiale et le FMI que par les disciplines de l'OMC. La différence entre « droits consolidés » $(\mathrm{OMC})$ et « droits 
réels » (issus de l'ajustement structurel) sont de l'ordre de $98 \%$ pour les Pays les moins avancés (PMA) contre $10 \%$ en moyenne pour les pays riches. Cependant, les raisons de la non-utilisation de cette importante marge de manœuvre existante pour les PMA ne sont pas évoquées. Ce débat renvoie à l'impossibilité de cette mise en œuvre de politiques commerciales, et sur le plan théorique au dépassement du concept de sécurité alimentaire vers celui de souveraineté alimentaire. Ce dernier inclut en effet le droit à la protection, seul instrument à la disposition de pays ne disposant pas des ressources nécessaires à un soutien massif. Cet instrument apparaît, paradoxalement, comme la forme de soutien au secteur agricole la moins protectionniste.

La distinction généralement admise entre les expressions de " sécurité alimentaire " (food security) et de "sécurité sanitaire des aliments " (food safety) n'est pas été retenue par les auteurs. La définition de la sécurité alimentaire qu'ils retiennent est une approche plus globale qualifiée de postmoderne : "État caractérisant un pays capable d'assurer une alimentation saine et équilibrée ". Cette approche présente l'inconvénient de masquer la situation spécifique des PFRDV, pays pour lesquels la disponibilité des aliments, la stabilité des approvisionnements et l'accès par tous aux denrées reste les composants fondamentaux de la sécurité alimentaire.

À partir de cette approche nouvelle, l'ouvrage traite des risques et crises liés à la qualité des aliments. De la même manière, les " politiques publiques de sécurité alimentaire " se concentrent sur les aspects sanitaires et nutritionnels des politiques. La question essentielle des politiques et stratégies de sécurité alimentaire des pays ou, la " gravité de la faim » selon l'expression de la FAO, son intensité est la plus grande, reste centrale. L'objectif principal dans ce cas reste le renforcement des capacités de production et d'accès aux biens alimentaires dans les nombreux PFRDV qui ont, pour la plupart, la capacité physique et économique de pourvoir à leurs besoins. La volonté politique d'élaboration et de mise en œuvre de véritables stratégies globales, alliant rôle du marché et de l'État, croissance de la production et satisfaction des besoins domestiques devient alors prioritaire dans la lutte contre l'insécurité alimentaire. La faim résulte de l'impossibilité de produire ou d'acheter les denrées alimentaires nécessaires à la vie, et le milliard de personnes concerné n'est pas constitué majoritairement de consommateurs qui n'auraient pas assez d'argent pour acheter leur nourriture, mais de producteurs de produits agricoles et de denrées alimentaires. Près de $75 \%$ d'entre eux vivent dans des zones rurales et la plupart dépendent directement ou indirectement de l'agriculture. Les $25 \%$ restants sont urbains, souvent des paysans pauvres récemment condamnés à l'exode rural. La petite agriculture familiale est très majoritairement composée d'exploitations agricoles $d$ 'une superficie de moins de deux hectares et la majorité de ces petits paysans et des travailleurs agricoles sont des acheteurs nets de denrées alimentaires et vivent avec moins de 2 dollars par jour. Cet « étranglement de la masse des petits paysans » selon l'expression d'Olivier De Schutter est au cœur des problématiques de développement et des stratégies de sécurité alimentaire qui en forment le noyau.

Au-delà de ces choix thématiques et des limites qu'ils imposent quant à l'analyse des questions de sécurité alimentaire dans les pays les plus pauvres, l'ouvrage représente une contribution remarquable par la diversité des outils et des approches disciplinaires mobilisées, à l'analyse systémique de l'économie alimentaire dans sa complexité.

Gérard AZOULAY Université de Paris Sud 11 Faculté Jean-Monnet 
Michel Petit

\section{Pour une agriculture mondiale, productive et durable}

Éditions Quæ, collection Essais, Paris, 2011, 112 p., $13 €$

$D_{2072}$ epuis l'envolée des prix agricoles de 2007-2008, une question anime les agronomes, les économistes, ainsi que certaines organisations internationales comme la FAO. Comment nourrir la population mondiale sur un horizon à peu près convergent pour tout le monde, à savoir 2050 ? On aura remarqué deux choses: d'une part que chacun y allait de son estimation, de son exercice de prospective, tout en essayant d'y adjoindre quelques éléments de réponse plus ou moins fiables selon les sources et, d'autre part, que l'observateur/lecteur croule du même coup sous une abondante littérature traitant de cette question. Cette littérature a au moins l'immense mérite de faire réfléchir et de se forger une opinion sur l'état de la sécurité - ou plutôt de l'insécurité alimentaire dans le monde.

Le dernier ouvrage de Michel Petit entend livrer une contribution à ce débat, mais selon un angle de vue bien précis, celui de la science, de la technologie et, disons-le, de la croyance fervente en la continuité de ce que l'on appelle, pour aller vite, le productivisme. Partant du constat selon lequel l'agriculture moderne a certes participé activement à l'élévation des performances en matière de production d'aliments, l'auteur prend la pleine mesure des externalités négatives qui ont été occasionnées par plusieurs décennies de croissance agricole. Mais, et c'est la thèse centrale du livre, Michel Petit indique avec force de conviction, que, au regard des perspectives démographiques, d'urbanisation, de croissance des niveaux de vie, I'on ne saurait "sacrifier, pour l'avenir, le progrès de la productivité sur l'autel de la durabilité ". II s'ensuit que seul un cheminement vers ce que l'on appelait il y a encore peu de temps une " agriculture raisonnée ", constituerait une issue souhaitable et souhaitée. En d'autres termes, l'auteur invite le lecteur à partager une réflexion sur une nouvelle phase de la modernisation de l'agriculture et des systèmes de production.

L'ouvrage de Michel Petit s'organise autour de trois parties. La première se propose de dresser un bilan de la modernisation des structures agricoles initiée après la Seconde Guerre mondiale. Il est à cet égard et d'emblée rappelé que le combat contre la rareté est non seulement une donnée de base dans l'histoire humaine, mais que, surtout, les experts de l'époque se demandaient bien comment I'humanité allait procéder pour satisfaire ses besoins alimentaires. Cette perspective malthusienne - qui était d'ailleurs partagée par son adversaire, David Ricardo, hanté qu'il était par le spectre de l'état stationnaire du fait de l'existence, en agriculture notamment, de la loi des rendements décroissants - fut, on le sait, battue en brèche par les progrès accomplis par les agricultures à partir des années cinquante.

Toute la première partie de l'ouvrage consiste donc à rappeler que les productions agricoles, et plus spécifiquement la production de céréales ont, au cours des décennies écoulées, connu une croissance régulière apte à combler les besoins des populations. Michel Petit insiste sur la hausse des rendements céréaliers, laquelle a permis un doublement de la production céréalière mondiale. L'enseignement qu'en tire l'auteur, et cela à plusieurs reprises, est que la clé de la progression des productions céréalières réside dans la productivité. Pour le dire de manière sans doute un peu plus directe, c'est de la science que viendra la durabilité de l'agriculture de demain.

La croissance des rendements rencontre toutefois des limites. On sait en effet que, et Michel Petit nous le rappelle dans la 
première partie de son livre, les rendements plafonnent dans certaines régions du monde, posant le problème de la capacité de l'agriculture à nourrir la planète dans les deux ou trois décennies à venir. Ce constat conduit à dépasser la seule "productivité partielle ", que l'auteur assimile aux rendements, afin de regarder d'une part l'évolution de la " productivité totale " des facteurs de production (travail, capital, intrants), et d'autre part de mesurer les tendances des autres productions agricoles. II est du même coup procédé, dans une seconde partie, à un découpage géographique des tendances de la production et du profil des productivités totales. L'Asie et l'Amérique latine s'en tirent manifestement mieux que les anciennes puissances industrialisées ayant accompli leur « révolution agricole » après la guerre. Le chapitre consacré à l'Afrique et à son agriculture souligne le contraste avec les réussites sud américaines et asiatiques. Si des progrès ont été enregistrés en matière de production agricole des pays d'Afrique, ils sont restés mineurs. Michel Petit dresse à cette occasion un réquisitoire - qui semblera sévère aux yeux de certains lecteurs - des politiques menées depuis l'époque coloniale mais aussi depuis les indépendances, en particulier dans le domaine de la recherche agronomique.

Les performances du Brésil et de la Chine sont de ce fait appréhendées à l'aune des investissements publics réalisés dans la recherche agronomique. Les exemples retenus par Michel Petit, Brésil, Chine et Inde dans le cas du coton $B t$, constituent pour lui l'illustration que c'est dans une conduite fine et adaptée du progrès technique et agronomique que se situe le moteur de la croissance agricole de demain. Mais les trois exemples retenus par l'auteur débouchent très rapidement sur un vibrant plaidoyer en faveur des Organismes génétiquement modifiés (OGM), lesquels font d'ailleurs l'objet d'un chapitre entier dans la troisième et der- nière partie du livre, dédiée à une tentative de clarification de quelques débats importants, dont celui des OGM. II convient de s'y arrêter dans la mesure où ce chapitre ne manquera pas de prolonger un conflit particulièrement vif entre les partisans des OGM, dont fait partie Michel Petit, et leurs opposants. En tant qu'agroéconomiste, Michel Petit procède à une évaluation des coûts et des avantages des OGM. Or les avantages (réduction de I'usage des pesticides, hausse des rendements à l'hectare...) apparaissent, selon lui, supérieurs aux coûts supposés de ces OGM. S'agissant du risque de voir les firmes multinationales occuper des positions de monopole sur le marché des semences OGM, I'auteur semble faire confiance aux dispositifs de politiques publiques qui, à l'instar de la législation américaine, peut limiter ce pouvoir de monopole. On sait pourtant que de telles lois sont aisément détournables et contournées la plupart du temps, par le jeu notamment de filiales écran ou de prises de participation conduisant à la formation de minorité de blocage dans les conseils d'administration.

En matière de risque pour la santé humaine, il est dit que rien n'avait pu jusqu'à présent être démontré, ce qui apparaîtra pour le moins un peu lapidaire. De même, sur la question de la " contamination génétique ", en dépit de sa reconnaissance par la communauté scientifique, Michel Petit émet quelques doutes sur la solidité de ces résultats, limitant son propos au problème du délai de la généralisation de la contamination. Enfin, le regard porté sur les conséquences sociales du recours aux OGM, à savoir l'éviction des exploitants agricoles familiaux dans les pays les plus pauvres, suscitera là aussi la controverse, la position de Michel Petit étant de préconiser une " intégration dans le processus de modernisation des petites exploitations "(page 107), luimême fondé sur le progrès technique. Arrêtons-nous justement un instant sur ce vocable « intégration ». 
Le terme d' « intégration " a son importance et il convient de lire le propos de Michel Petit entre les lignes. Si la science et la technologie constituent les supports du devenir de l'agriculture, ce devenir passera manifestement par une intégration de l'agriculture familiale dans cette nouvelle phase de la modernisation, perspective qui n'est pas sans rappeler les anticipations des marxistes à propos de la question agraire, au sujet desquelles beaucoup d'encre avait coulé dans les années soixante-dix. Sur ce point, il a souvent été mis en avant le rôle des industries de la transformation dans le processus d'intégration des agriculteurs. Aujourd'hui, au regard des recommandations d'un agroéconomiste comme Michel Petit, le processus d'intégration se poursuit avec l'implication de plus en plus forte des firmes situées en amont de l'exploitant, dans le contrôle de cet exploitant. Bref, une agriculture subsumée sous les forces du capital. En un certain sens, l'ouvrage nous offre une belle occasion de rouvrir un débat qui, pour certains, apparaîtra comme anachronique, inutile, mais qui n'a pourtant jamais été autant d'actualité. Pour que l'agriculture soit performante en même temps que durable, elle doit se plier aux exigences du capitalisme. Et, depuis les travaux du philosophe Jürgen Habermas, on sait que le développement de la science dans un tel système renvoie à des dimensions idéologiques bien précises, sur lesquelles l'auteur fait, forcément, l'impasse.
Sans aller aussi loin dans la critique, on indiquera, pour finir, que les effets de la science sur la production agricole n'ont pas toujours les effets escomptés. Dans le cas de la Chine, si de réels progrès ont été accomplis depuis la fin des années soixante-dix, il n'en demeure pas moins qu'à ce jour, la Chine forme une économie très largement dépendante de l'extérieur pour certains de ses approvisionnements en nourriture, ainsi qu'en témoignent ses importations d'oléagineux et de maïs destinés à l'alimentation animale, voire aux biocarburants. $\mathrm{Si}$ le progrès technique était si efficace en Chine, comment s'expliquerait alors que cette nouvelle puissance pratique l'accaparement des terres agricoles - I'Afrique étant particulièrement exposée aux appétits de la Chine - afin de sécuriser ses approvisionnements alimentaires ? Le facteur scientifique, aussi important soitil dans l'accroissement des productions, ne saurait effacer la composante géopolitique. L'agriculture étant bien, de ce point de vue, en première ligne, au même titre que les matières premières énergétiques. Quoi qu'il en soit, un livre à ne pas négliger, tant il est porteur d'éléments de débat.

Thierry POUCH

Pôle économie et politiques agricoles de I'APCA et laboratoire Organisations marchandes et Institutions Université de Reims Champagne-Ardenne 